\title{
A Simple Rapid Method for Measuring Liver Steatosis Using Bioelectrical Impedance
}

\author{
TOMOKO YOSHIMOTO-HARAMURA ${ }^{1 *}$, TAKANOBU HARA ${ }^{1 *}$, AKIHIKO SOYAMA ${ }^{1}$, \\ TOTA KUGIYAMA ${ }^{1}$, HAJIME MATSUSHIMA $^{1}$, KUNIHITO MATSUGUMA $^{1}$, \\ HAJIME IMAMURA $^{1}$, TAKAYUKI TANAKA ${ }^{1}$, TOMOHIKO ADACHI ${ }^{1}$, MASAAKI HIDAKA ${ }^{1}$, \\ SHUICHI OKABE ${ }^{2}$, MASAKAZU MURATA ${ }^{3}$ and SUSUMU EGUCHI ${ }^{1}$ \\ ${ }^{1}$ Department of Surgery, Nagasaki University Graduate School of Biomedical Sciences, Nagasaki, Japan; \\ ${ }^{2}$ Yamato Scale Co., Ltd, Commercial Weighing Solutions Division, Hyogo, Japan; \\ ${ }^{3}$ The Graduate School of Fisheries and Environmental Sciences, Nagasaki University, Nagasaki, Japan
}

\begin{abstract}
Background/Aim: Easy measurement of liver steatosis without pathological diagnosis may help improve donor surgery efficiency and increase the chances of organ donations. We analyzed the correlations between bioelectrical impedance (BI) in human livers, liver fat content, and pathological findings. Materials and Methods: Sixteen tumor-free liver specimens resected during elective oncological surgery were analyzed. All samples were stored in ice chilled saline before BI measurement. The BI measurement was performed using a device with the tetrapolar circuit method in which the current and voltage electrodes are independent. Liver cholesterol and triglyceride levels were investigated from the same specimen using the Soxhlet extraction method. Pathological findings were examined by counting the number of hepatocytes with fatty changes per high-power field. Results: The median liver steatosis percentage was $0.4 \%$. The liver steatosis percentage was significantly correlated with the intrahepatic triglyceride content $(r=0.82, p<0.001)$. Linear regression of the measurements and predicted values yielded an $r^{2}$ of 0.63 between the BI at $100 \mathrm{kHz}$ and liver steatosis, indicating reasonable agreement $(p<0.001)$. Conclusion: BI analysis is a simple, non-invasive method that can be easily applied to evaluate liver steatosis.
\end{abstract}

This article is freely accessible online.

*These Authors contributed equally to this study.

Correspondence to: Susumu Eguchi, MD, Ph.D., Department of Surgery, Nagasaki University Graduate School of Biomedical Sciences, 1-7-1 Sakamoto, Nagasaki, 852-8501, Japan. Tel: +81 958197316, Fax: +81 958197319, e-mail: sueguchi@nagasaki-u.ac.jp

Key Words: Liver steatosis, bioelectrical impedance, liver transplantation, graft evaluation.
Organ shortages for liver transplantation and the increasing mortality of patients on the transplant waiting list have led to the use of expanded criteria donor (ECD) livers to expand the donor pool. Among these ECD livers, hepatic steatosis is one of the most frequent diseases (1). The gold standard for evaluating hepatic steatosis is a histological analysis by a pathologist. The fatty liver degree is considered mild when the percentage of fatty vacuoles in the hepatocytic cytoplasm is $<30 \%$, moderate when the percentage is between $30 \%$ and $60 \%$, and severe when the percentage is $\geq 60 \%$ (2).

The success of deceased donor liver transplantation depends on graft viability. Previous reports indicate a high frequency of primary nonfunction of the graft in severe macrovesicular steatosis $(3,4)$. Moderate to severe macrovesicular steatosis is considered as a major cause of severe liver preservation injury (5). Therefore, assessing donor liver steatosis during procurement surgery is a difficult task for the transplant team. Donor surgeons perform the initial evaluation during procurement surgery via visual inspection and palpation. However, the criteria for determining the graft color and texture are subjective and rely solely on the experience of the procurement surgeon (1). Rey et al. reported that neither preoperative ultrasound assessments nor gross assessments at the time of procurement surgery are reliable for assessing liver steatosis (6). Diagnostic imaging, such as computed tomography and magnetic resonance imaging, can help assess liver steatosis more objectively, but such information is rarely available for deceased donors. The next step is to perform a liver biopsy for microscopic examination. Because of the heterogeneous fat distribution in the liver, performing two or more biopsies from different segments can yield more accurate information (6). However, a previous report indicated that the positive predictive values of macroscopic evaluation at the time of procurement were $71 \%$ for severe, $46 \%$ for moderate, and only $17 \%$ for mild steatosis (7). Thus, microscopic 


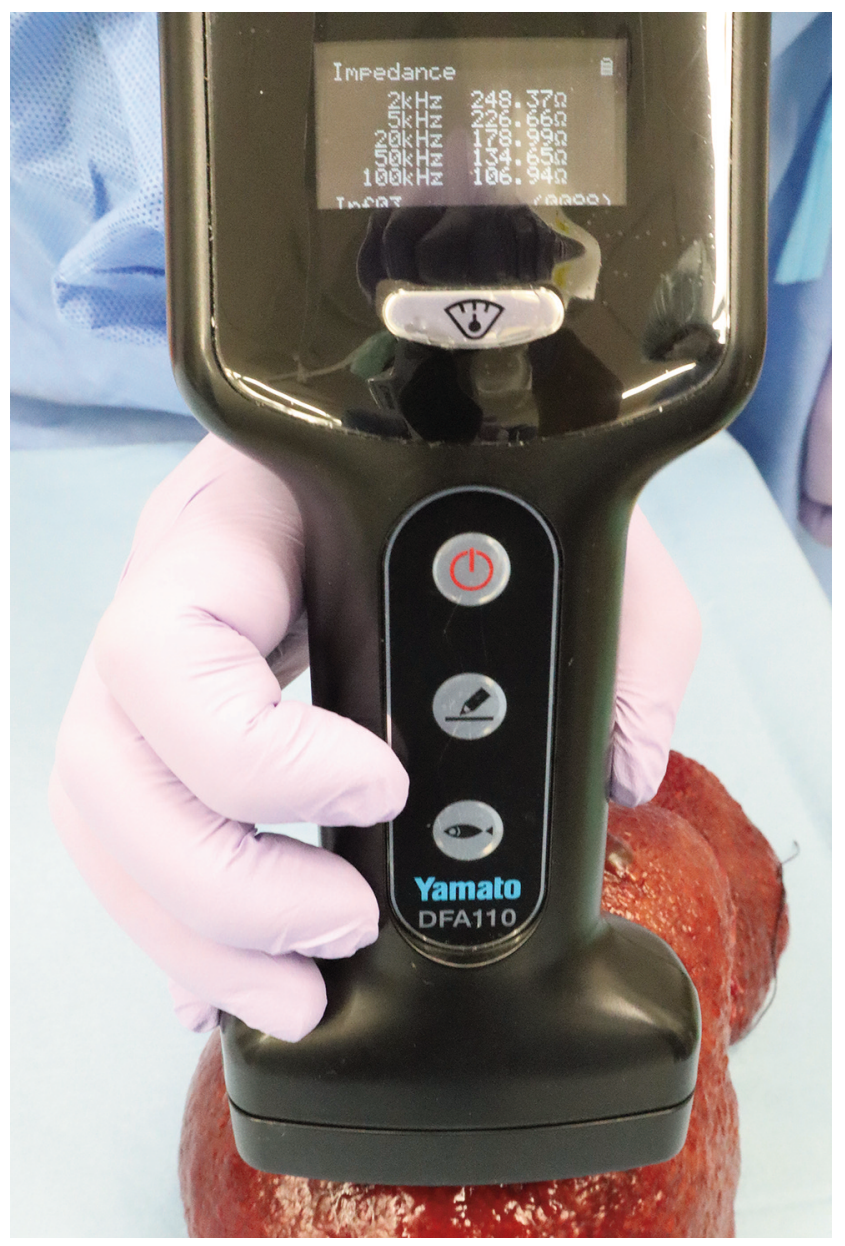

Figure 1. Measurement of bioelectrical impedance values on the liver surface after cold storage.

examination remains the gold standard, but is problematic because it damages the graft, enables analogizing only the fat content of the whole liver with a few specimens, and takes a long time to diagnose. Additionally, pathologists may be unavailable for some procurement surgeries.

Bioelectrical impedance (BI) analysis is a non-invasive, safe and simple method of evaluating body composition by applying a weak alternating current to the body. The body composition analyzer estimates fat and muscle mass based on the impedance value, and calculates the value by adding the patient's height, weight, age, and sex to the impedance value. This value is used to evaluate body composition and nutritional status. Electric currents can pass through fat-free masses, which are rich in water and electrolytes, but cannot pass through adipose tissue, which contains very little water (8). In large amounts of adipose tissue, the current flow becomes difficult and the electrical resistance value (impedance) increases.
Measuring liver BI using a similar technique may enable estimating the degree of fatty liver. Non-invasively and easily measuring the degree of fatty liver may help in organ retrieval by allowing repeated measurements and determining the heterogeneous fat distribution in the liver. Previous studies using animal models have reported good correlations between BI measurements and liver steatosis (9-11). Here, we evaluated whether BI could be used to evaluate the rate of liver steatosis in human livers in a pilot study of non-cirrhotic patients who underwent a hepatectomy.

\section{Materials and Methods}

Materials. Sixteen of 107 patients who underwent hepatectomies beyond segmental resection at our hospital from January 2016 to December 2017 provided written consent and were included in the study. Approximately $30 \mathrm{~g}$ of noncancerous liver tissue was collected from liver resection specimens removed from these patients. The collected specimens were stored in ice at $4^{\circ} \mathrm{C}$ for at least $20 \mathrm{~min}$ to standardize the temperature conditions for impedance measurement. The institutional review board of Nagasaki University approved the study (approval number: 15072754-2).

BI analysis. BI analysis was performed using a Fish analyzer DFA 100 (Yamato Scale Co., Ltd., Hyogo, Japan; Figure 1). This device uses the tetrapolar circuit method, where the current and voltage electrode are independent, and the electrode structure does not affect the impedance. The electrodes are all $1 \mathrm{~cm}$ apart; an alternating $350-\mu \mathrm{A}$ current is applied from the outer current electrode, and the voltage is detected at the inner voltage electrode. The detected voltage is amplified by the amplifying circuit, converted to direct current by the rectifying and smoothing circuits, and finally converted to a digital signal by the analog/digital converter circuit. The central processing unit then calculates the impedance. The device also uses a multifrequency measurement method that allows electricity to flow at multiple frequencies (e.g., 2, 5, 20, 50, and $100 \mathrm{kHz}$ ).

Quantification measurement of lipids in the liver. Intrahepatic lipids were measured at Skylight Biotech, Inc. (Akita, Japan). After analyzing the BI of the resected liver, approximately $1 \mathrm{~g}$ of liver tissue was used for measurements. Lipids were extracted via the Folch method (12). Triglycerides (TGs) and total cholesterol (T$\mathrm{CHO}$ ) were quantified in $\mathrm{mg} / \mathrm{g}$ using the Cholestest $\mathrm{TG}$ and Cholestest CHO (Sekisui Medical, Tokyo, Japan), respectively.

Evaluation of liver steatosis. Formalin-fixed, paraffin-embedded liver sections were stained with hematoxylin and eosin (Figure 2). The prepared histological slides were scanned and photographed using a BZ-X810 microscope (Keyence, Osaka, Japan). The proportion of fatty change was analyzed using BZ-H4 software (Keyence). The area of fatty change per field of view was measured at $100 \times$ magnification, and the percentage of liver steatosis was calculated. The average of five fields of view per sample was calculated.

Statistical analysis. IBM SPSS Statistics 26 (IBM Corp., New York, NY, USA) was used for statistical analysis. A Mann-Whitney $U$-test 

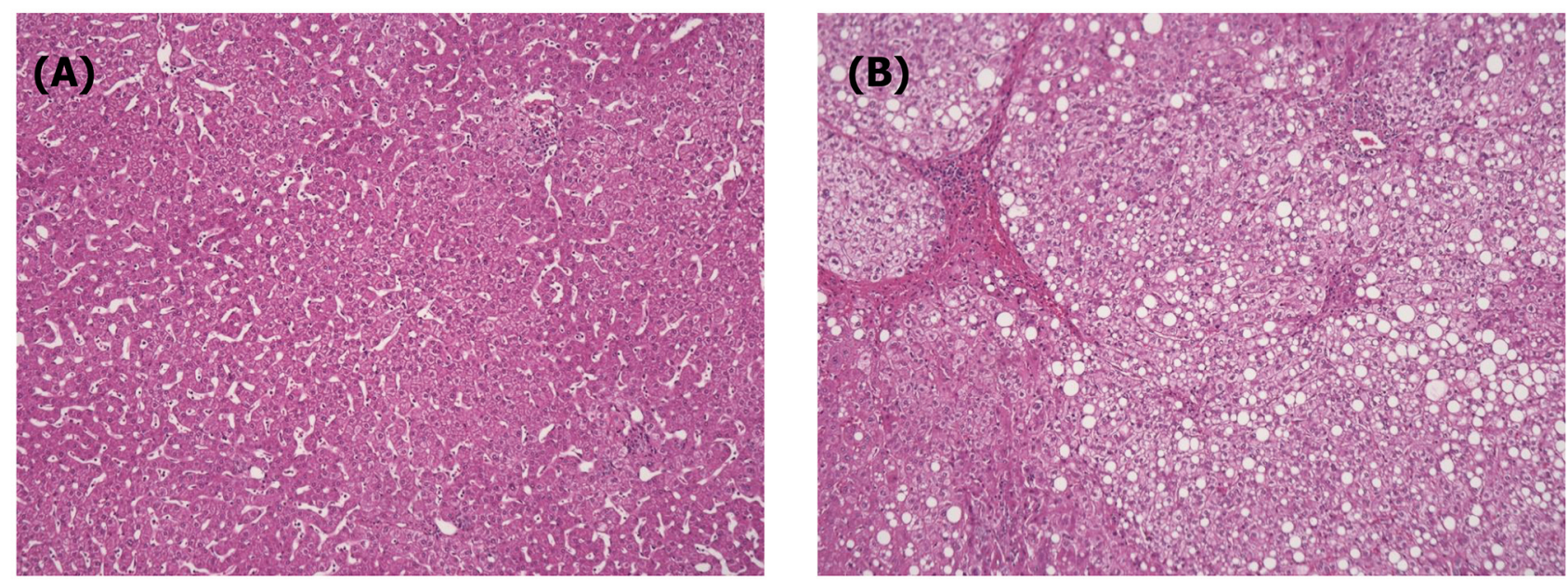

Figure 2. Hematoxylin-eosin-stained liver tissue. (A) $0.01 \%$ liver steatosis; (B) $8.0 \%$ liver steatosis.

was used to analyze continuous data, and a chi-square test was used for categorical data. Single regression analysis was used to assess the relationship between BI value and percentage of liver steatosis. $p$-Value $<0.05$ was considered statistically significant.

\section{Results}

Patient characteristics. Table I shows the patients' background characteristics. Sixteen patients (nine women and seven men) were enrolled. The median age was 72 years (range $=24-83$ years); the median body mass index was 24.4 (range $=17.3$ 30.7). Six patients underwent surgery for hepatocellular carcinoma, five for intrahepatic cholangiocarcinoma, four for colorectal liver metastases, and one for hepatolithiasis. Seven patients had preexisting hypertension, three had diabetes, and one had hyperlipidemia. The median preoperative laboratory values were albumin: $4.05 \mathrm{~g} / \mathrm{dl}$ (range=2.8-5.0 g/dl), bilirubin: $0.9 \mathrm{mg} / \mathrm{dl}$ (range=0.3-1.8 g/dl), AST: $20.5 \mathrm{U} / \mathrm{l}$ (range=13-75 $\mathrm{g} / \mathrm{dl}$ ), ALT: $20.5 \mathrm{U} / 1$ (range=9-119 g/dl), and PT INR: 1.1 (range $=0.9-1.2 \mathrm{~g} / \mathrm{dl})$.

Relationship between amount of intrahepatic lipids and percentage of liver steatosis. The median intrahepatic T-CHO content was $0.3 \mathrm{mg} / \mathrm{g}$ (range=0.2-0.5 mg/g); the median intrahepatic TG content was $1.0 \mathrm{mg} / \mathrm{g}$ (range=0.3-11.5 $\mathrm{mg} / \mathrm{g})$. The median percentage of liver steatosis was $0.4 \%$ (range $=0.01 \%-8.1 \%$ ). Figure $3 \mathrm{~A}$ and $\mathrm{B}$ shows the relationship between these parameters and the percentage of liver steatosis. The percentage of liver steatosis was significantly correlated with the intrahepatic TG content $(\mathrm{r}=0.82, p<0.001)$.

Relationship between BI values and percentage of liver steatosis. The median BIs were $324.5 \Omega$ (range=179.2-600.7
Table I. Patient characteristics.

\begin{tabular}{lc}
\hline Patients (n) & 16 \\
Gender, male & $7(43.8 \%)$ \\
Age & $72[24-83]$ \\
Body mass index & $24.4[17.3-30.7]$ \\
Indication for surgery & $6 \mathrm{HCC} / 5 \mathrm{ICC} / 4$ CRLM/1 Hepatolithiasis \\
Comorbidities & \\
Hypertension & $7(43.8 \%)$ \\
Diabetes mellitus & $5(31.3 \%)$ \\
Hyperlipidemia & $1(6.2 \%)$ \\
Comorbidities & $7(43.8 \%)$ \\
Hypertension & $5(31.3 \%)$ \\
Laboratory data & \\
Albumin (g/dl) & $4.05[2.8-5.0]$ \\
Total bilirubin (mg/dl) & $0.9[0.3-1.8]$ \\
AST (U/l) & $22.5[13-75]$ \\
ALT (U/l) & $20.5[9-119]$ \\
PT-INR & $1.1[0.9-1.2]$ \\
\hline
\end{tabular}

HCC: Hepatocellular carcinoma; ICC: intrahepatic cholangiocarcinoma; CRLM: colorectal liver metastasis; AST: aspartate aminotransferase; ALT: alanine aminotransferase; PT-INR: international normalized ratio of prothrombin time. Data are represented as $\mathrm{n}(\%)$ or median [range].

$\Omega)$ at $2 \mathrm{kHz}, 290.9 \Omega($ range $=173.0-517.0 \Omega)$ at $5 \mathrm{kHz}, 216.5$ $\Omega($ range $=155.6-344.7 \Omega)$ at $20 \mathrm{kHz}, 160.4 \Omega($ range $=123.3-$ $259.0 \Omega$ ) at $50 \mathrm{kHz}$, and $127.5 \Omega($ range=98.6-201.6 $\Omega$ ) at $100 \mathrm{kHz}$. Figure 4 shows the relationship between the BI values measured at $2,5,20,50$, and $100 \mathrm{kHz}$ and the percentage of liver steatosis. Linear regression of the measurements and predicted values yielded an $r^{2}$ of 0.63 between the BI at $100 \mathrm{kHz}$ and liver steatosis, indicating reasonable agreement $(p<0.001)$. Based on this curve, the percentage of liver steatosis could be determined by the formula: [0.054×(liver BI value at $100 \mathrm{kHz})-6.0832$ ]. 
A

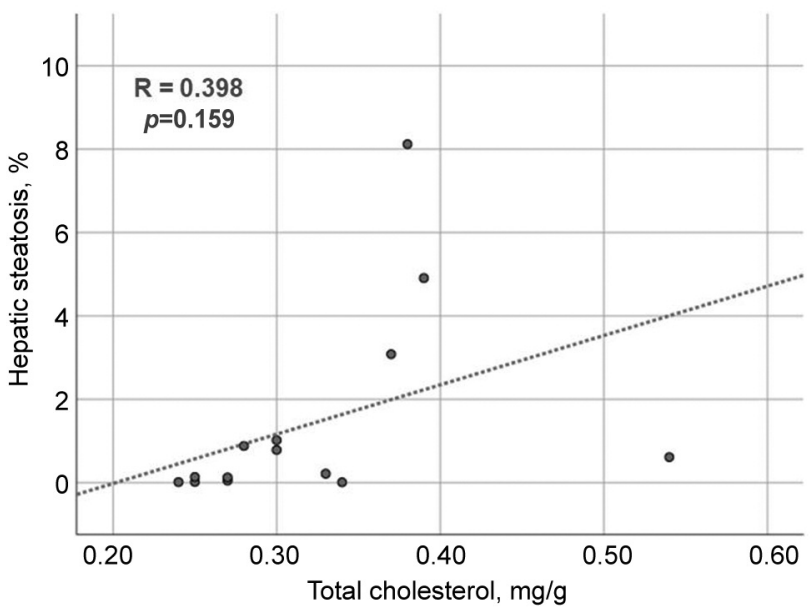

B

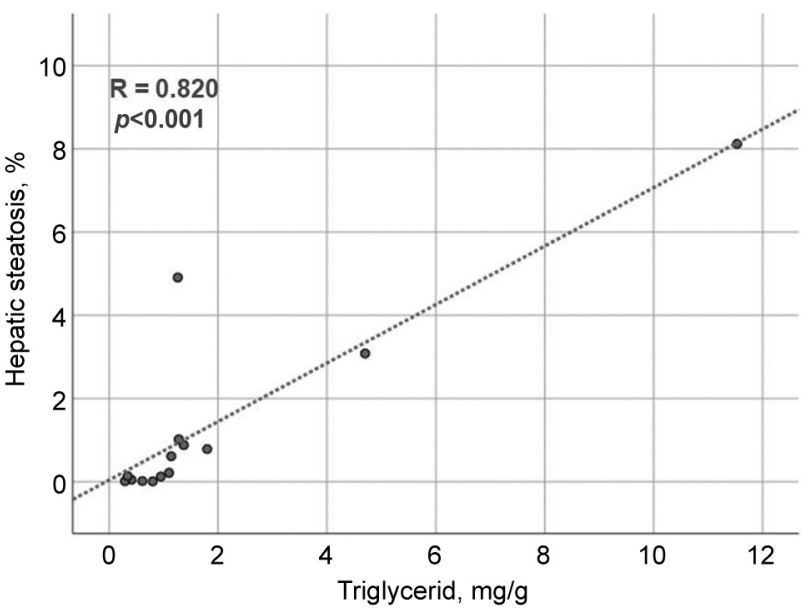

Figure 3. Relationship between percentage of liver steatosis and amount of intrahepatic lipids. (A) Relationship between percentage of liver steatosis and intrahepatic total cholesterol content; $(B)$ relationship between percentage of live steatosis and intrahepatic triglyceride content.
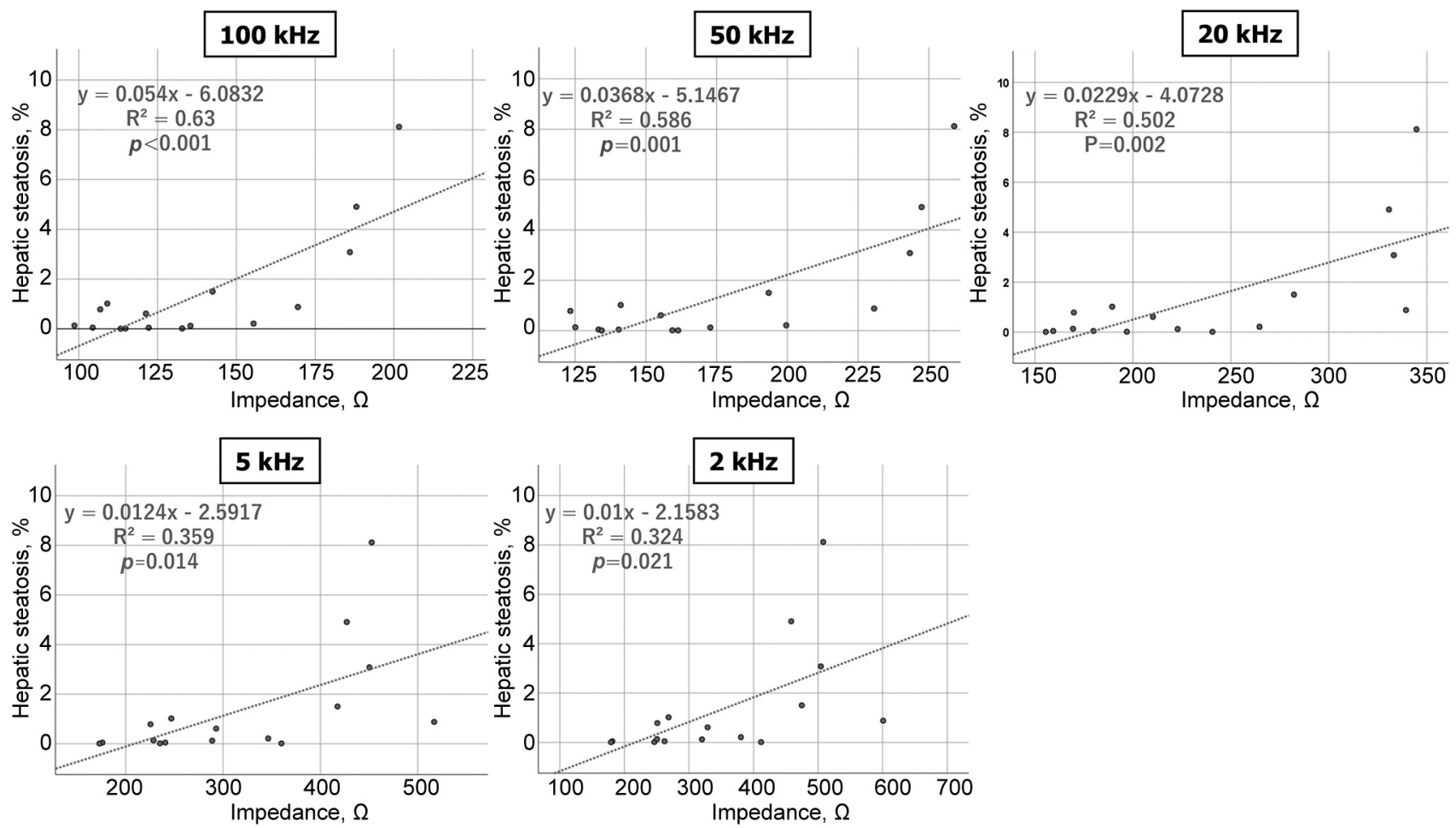

Figure 4. Relationship between percentage of liver steatosis and liver BI at multiple frequencies.

\section{Discussion}

Here, we showed that the liver BI measurement could predict the degree of liver steatosis. Liver steatosis is considered a major risk for primary graft nonfunction after liver transplantation. This risk should be eliminated as much as possible. Histopathological examination at the time of donor surgery is the gold standard for evaluating liver steatosis. However, it is not available 24 hours a day at all facilities, and the evaluation is subjective among pathologists. Additionally, 
multiple biopsies are required to evaluate the entire liver, which causes minor but significant graft damage. Therefore, a better method is needed to measure liver steatosis.

The BI method is simple, fast, can be measured with a small portable device and allows objective evaluation by quantification. However, to date, few studies have reported liver steatosis evaluations using the BI method. Hwang et al. measured the total body fat composition in 302 patients using a body fat analyzer via the BI method. The measurements were performed with patients in the supine position using a bipolar electrode configuration placed on the dorsa of both hands. The BI-derived fat composition was calculated using age, sex, height, and weight. The results were compared with the histopathological degree of liver steatosis determined from the biopsy. The results showed a poor relationship between the two parameters $\left(r^{2}=0.14, p<0.01\right)$; the authors concluded that predicting the degree of fatty liver by measuring total body composition is difficult using the BI method alone (13). Parramon et al. (2007) demonstrated that multifrequency tissue $\mathrm{BI}$ measurements could be used to detect liver steatosis in vivo (9). In 2009, these authors examined the relationship between low-frequency BI and histopathological degree of liver steatosis using a rat-liver model. As in our study, they used a BI probe consisting of four electrodes. They applied a $50-\mu \mathrm{A}$ alternating current, measured the resulting potential across the inner electrodes, and analyzed the measurement at $1 \mathrm{kHz}$. The BI measurements correlated well with the percentage of liver macrosteatosis (Pearson correlation coefficient: 0.73). Thus, their study showed that direct BI measurements of the liver may predict liver steatosis. Bhati et al. (2009) performed BI analysis using human livers to evaluate macrovesicular steatosis. They used the Maltron BI analyzer (BioScan 915, Maltron International Ltd., London, UK) with tetrapolar electrodes. The recorded resistance and impedance were compared with the histopathological grade of the biopsied liver steatosis. Their results showed that the presence of macrovesicular steatosis correlated with resistance $(p=0.03)$. However, the impedance and macrovesicular fat were not correlated ( $p=0.08$ ) (14). This result differed from our results, likely because the measurements were taken only at $50 \mathrm{kHz}$ and intraoperatively, while hepatic blood flow was maintained.

In the present study, the results were less susceptible to local factors and background noise because we measured the BI while the livers were ice-cold on a back table. We thought that body temperature and blood flow might affect the results Donor liver grafts are kept ice-cold during transport; thus, our measurement conditions were reasonable. We also measured the BI values using various frequencies and searched for the condition that best correlated with liver steatosis. We examined frequencies ranging from $2-100 \mathrm{kHz}$, and the readings at 100 $\mathrm{kHz}$ were the best predictors. To our knowledge, this is the first study to show an association between directly measured liver $B I$ values and the degree of liver steatosis.
This study had several limitations. First, the study was designed to be a pilot clinical trial, and the number of cases was limited. Additionally, the number of patients with advanced fatty liver was small because we included patients who underwent hepatic resection with more than a sectionectomy, and many had preserved liver function. Finally, we measured the BI with only a single device; thus, validation is necessary.

Interestingly, a previous report indicated a relationship between changes in BI values during cold preservation and ischemic damage to kidney grafts. A possible explanation may be that cellular edema narrows the extra cellular space and reduces the width of the electrical path for lowfrequency currents (15). BI analysis may help assess both liver steatosis and graft freshness after a relatively long warm ischemic time in donation and circulatory death.

In conclusion, BI is a simple, non-invasive and convenient method that may help clinicians evaluate liver steatosis. Although further research is needed, the BI method may become the new gold standard for graft evaluation of liver steatosis during donor surgery.

\section{Funding}

This work was supported by the Japan Society for the Promotion of Science, Tokyo, Japan (JSPS) KAKENHI Grant Number JP16K19900. The financial supporter had no role in study design, data collection and analysis, decision to publish, or preparation of the manuscript.

\section{Conflicts of Interest}

The Authors declare no conflicts of interest in relation to this study.

\section{Authors' Contributions}

TYH and TH drafted and revised the article. TYH, TH, and AS performed the experiment. TK was responsible for the histopathological diagnosis. HM, KM, HI, TT, and TA contributed to perioperative care. TH, AS and $\mathrm{MH}$ contributed to the study design. SO made adjustments and gave advice on the use of the devise. MM and SE supervised the writing of the manuscript. All Authors approved the submitted version of the manuscript.

\section{Acknowledgements}

The Authors thank Traci Raley, MS, ELS, from Edanz (https://jp.edanz.com/ac) for editing a draft of this manuscript.

\section{References}

1 McCormack L, Dutkowski P, El-Badry AM and Clavien PA: Liver transplantation using fatty livers: always feasible? J Hepatol 54(5): 1055-1062, 2011. PMID: 21145846. DOI: 10.1016/j.jhep.2010.11.004 
2 Nocito A, El-Badry AM and Clavien PA: When is steatosis too much for transplantation? J Hepatol 45(4): 494-499, 2006. PMID: 16919359. DOI: 10.1016/j.jhep.2006.07.017

3 Todo S, Demetris AJ, Makowka L, Teperman L, Podesta L, Shaver T, Tzakis A and Starzl TE: Primary nonfunction of hepatic allografts with preexisting fatty infiltration. Transplantation 47(5): 903-905, 1989. PMID: 2655230. DOI: 10.1097/00007890-198905000-00034

4 Rinella ME, Alonso E, Rao S, Whitington P, Fryer J, Abecassis M, Superina R, Flamm SL and Blei AT: Body mass index as a predictor of hepatic steatosis in living liver donors. Liver Transpl 7(5): 409414, 2001. PMID: 11349260. DOI: 10.1053/jlts.2001.23787

5 Briceño J, Padillo J, Rufián S, Solórzano $G$ and Pera C: Assignment of steatotic livers by the Mayo model for end-stage liver disease. Transpl Int 18(5): 577-583, 2005. PMID: 15819807. DOI: $10.1111 / \mathrm{j} .1432-2277.2005 .00091 . x$

6 Rey JW, Wirges U, Dienes HP and Fries JW: Hepatic steatosis in organ donors: disparity between surgery and histology? Transplant Proc 41(6): 2557-2560, 2009. PMID: 19715973. DOI: $10.1016 /$ j.transproceed.2009.06.121

7 Adam R, Reynes M, Johann M, Morino M, Astarcioglu I, Kafetzis I, Castaing D and Bismuth H: The outcome of steatotic grafts in liver transplantation. Transplant Proc 23(1 Pt 2): 15381540, 1991. PMID: 1989281.

8 Demura S, Sato S and Kitabayashi T: Percentage of total body fat as estimated by three automatic bioelectrical impedance analyzers. J Physiol Anthropol Appl Human Sci 23(3): 93-99, 2004. PMID: 15187381. DOI: 10.2114/jpa.23.93

9 Parramon D, Erill I, Guimerà A, Ivorra A, Muñoz A, Sola A, Fondevila C, García-Valdecasas JC and Villa R: In vivo detection of liver steatosis in rats based on impedance spectroscopy. Physiol Meas 28(8): 813-828, 2007. PMID: 17664674. DOI: 10.1088/0967-3334/28/8/005

10 Hessheimer AJ, Parramón D, Guimerà A, Erill I, Rimola A, García-Valdecasas JC, Villa $\mathrm{R}$ and Fondevila C: A rapid and reliable means of assessing hepatic steatosis in vivo via electrical bioimpedance. Transplantation 88(5): 716-722, 2009. PMID: 19741471. DOI: 10.1097/TP.0b013e3181b391c0
11 Gonzalo MA, Martínez-Beamonte R, Palacios P, Marín J, Castiella T, Surra J, Burdío F, Sousa R, Güemes A, Osada J and García-Gil A: Analysis of tissue bioimpedance as a measurement of liver steatosis: experimental model in large animals. Transplant Proc 44(6): 1579-1583, 2012. PMID: 22841219. DOI: $10.1016 / \mathrm{j}$.transproceed 2012.05 .006

12 Folch J, Lees M and Sloane Stanley GH: A simple method for the isolation and purification of total lipides from animal tissues. J Biol Chem 226(1): 497-509, 1957. PMID: 13428781.

13 Hwang S, Yu YD, Park GC, Park PJ, Choi YI, Choi NK, Kim KW, Song GW, Jung DH, Yun JS, Choi SY and Lee SG: Bioelectrical impedance analysis for evaluation of donor hepatic steatosis in living-donor liver transplantation. Transplant Proc 42(5): 1492-1496, 2010. PMID: 20620461. DOI: $10.1016 /$ j.transproceed 2010.03 .137

14 Bhati CS, Silva MA, Wigmore SJ, Bramhall SR, Mayer DA, Buckels JA, Neil DA, Murphy $\mathrm{N}$ and Mirza DF: Use of bioelectrical impedance analysis to assess liver steatosis. Transplant Proc 41(5): 1677-1681, 2009. PMID: 19545706. DOI: $10.1016 / \mathrm{j}$.transproceed 2009.01 .083

15 Genescà M, Ivorra A, Sola A, Palacios L, Goujon JM, Hauet T, Villa R, Aguiló J and Hotter G: Electrical bioimpedance measurement during hypothermic rat kidney preservation for assessing ischemic injury. Biosens Bioelectron 20(9): 18661871, 2005. PMID: 15681207. DOI: 10.1016/j.bios.2004.06.038

Received December 19, 2021

Revised January 9, 2022

Accepted January 10, 2022 